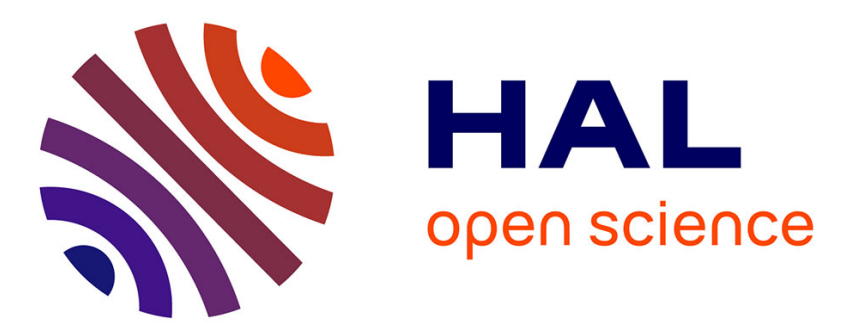

\title{
Design of solid core photonic bandgap fibers for visible supercontinuum generation
}

\author{
V. Pureur, J.M. Dudley
}

\section{To cite this version:}

V. Pureur, J.M. Dudley. Design of solid core photonic bandgap fibers for visible supercontinuum generation. Optics Communications, 2011, 284 (6), pp.1661-1668. 10.1016/j.optcom.2010.11.037 . hal-00582921

\section{HAL Id: hal-00582921 \\ https://hal.science/hal-00582921}

Submitted on 6 May 2021

HAL is a multi-disciplinary open access archive for the deposit and dissemination of scientific research documents, whether they are published or not. The documents may come from teaching and research institutions in France or abroad, or from public or private research centers.
L'archive ouverte pluridisciplinaire HAL, est destinée au dépôt et à la diffusion de documents scientifiques de niveau recherche, publiés ou non, émanant des établissements d'enseignement et de recherche français ou étrangers, des laboratoires publics ou privés. 


\title{
Design of solid core photonic bandgap fibers for visible supercontinuum generation
}

\author{
Vincent Pureur*, John M. Dudley \\ Institut FEMTO-ST, Département d'Optique P. M. Duffieux, CNRS UMR 6174, Université de Franche-Comté, 25030 Besançon, France
}

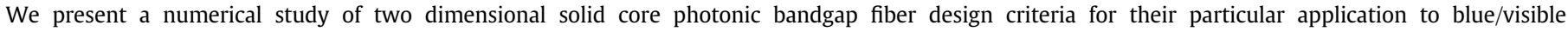

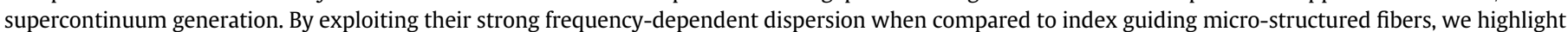

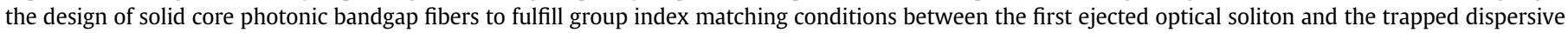

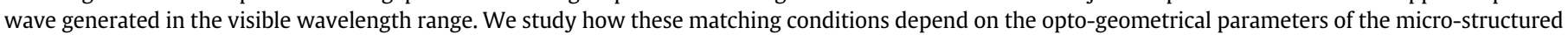

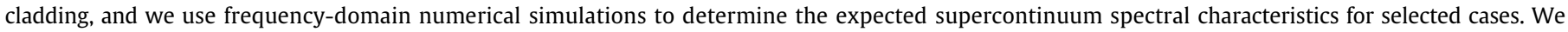

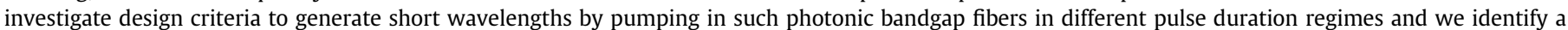

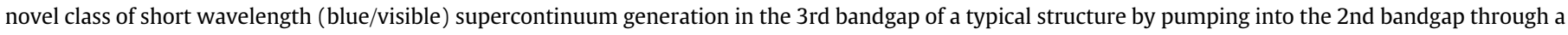
high attenuation spectral region.
\end{abstract}

\section{Introduction}

The generation of broadband supercontinuum spectra has attracted much interest over the last decade since the advent of photonic crystal fiber (PCF) [1-5]. Taking advantage of the novel dispersive and non linear properties [6], the generation of new frequencies through supercontinuum generation in PCF has found many applications in source development, optical coherence tomography, spectroscopy, confocal microscopy and optical frequency metrology.

An important area of the current activity is the study of techniques to extend the non linear spectral broadening further into the visible or the blue spectral range. One approach that has been extensively studied is the mechanism whereby a dispersive wave (DW) generated in the normal group velocity dispersion (GVD) regime is trapped by a soliton propagating in the infrared in the anomalous GVD regime such that there is enhanced frequency shifting to shorter wavelengths [7-10]. In order to achieve this, group index matching conditions must be fulfilled between the dispersive wave and the soliton, and this effect has been observed experimentally in the visible range in index guiding PCFs both in the picosecond and CW pumping regime pumping around $1060-1080 \mathrm{~nm}$ [11-13].

The extent of the supercontinuum in the infrared is limited in silica PCFs by the increase of $\mathrm{OH}^{-}$ions absorption beyond $2.4 \mu \mathrm{m}$ with a strong peak around $1.38 \mu \mathrm{m}$, limiting then the coupled energy to the trapped dispersive wave in the visible [13]. In order to improve short

\footnotetext{
* Corresponding author.

E-mail address: Vincent.Pureur@femto-st.fr (V. Pureur).
}

wavelength component generation in PCF, one way is to decrease water absorption around $1.38 \mu \mathrm{m}$ from typically $600 \mathrm{~dB} / \mathrm{km}$ to about $90 \mathrm{~dB} / \mathrm{km}$ by reducing surface contamination during the fabrication process through chemical cleaning [14]. However, another way is to modify the group index curve of the fundamental core guided mode by tailoring the geometry of the photonic crystal cladding and the core size and by keeping the zero dispersion wavelength (ZDW) close enough to the pump. Because the group index on the short wavelength side is mainly dominated by the material dispersion (silica), efforts have been recently made to optimize the waveguide group index in the infrared of index guiding PCFs to push the supercontinuum further into the near ultraviolet [13]. However in this case the optimization also shifts the ZDW further away from the pump, limiting the efficiency of the short wavelength generation in the quasi-CW regime [15].

In this work we consider how the novel guidance characteristics of two dimensional solid core photonic bandgap (PBG) fibers can overcome this limitation, and be used to generate short wavelengths via supercontinuum generation. In fact, recent experimental work has reported spectral broadening in PBG fibers in the fundamental bandgap using sub-nanosecond pulses at $1064 \mathrm{~nm}$ [16] but in the infrared from $1 \mu \mathrm{m}$ to $1.6 \mu \mathrm{m}$ and not in the visible. We show here that group index matching conditions can be found between dispersive waves in the blue/visible and optical solitons propagating in the infrared without reaching wavelength above $1 \mu \mathrm{m}$. In addition, motivated by recent numerical studies showing energy transfer between two different transmission bands across a spectral region of high attenuation [17], we also simulate trapped dispersive wave generation in the blue edge of the 3rd bandgap of a typical structure by pumping into the anomalous GVD regime of the 2nd transmission 
band in order to highlight the potential of PBG fibers to generate blue/ visible components.

\section{Solid core photonic bandgap fibers: group index matching}

Two dimensional solid core PBG fibers propagate light in a low index defect through a PBG guidance mechanism [18]. In order to obtain such an effect, high refractive index inclusions (Germanium/ fluorine doped-silica or oil-infiltrated air holes) are periodically embedded into a low index background made generally of pure silica as the core (see Fig. 1) [18]. This guidance mechanism involves propagation of the light through different spectral windows of different order (different PBGs) each associated with its own linear GVD and confinement losses and non linear properties; both the linear and non linear properties are strongly frequency-dependent [18].

According to the Anti Resonant Reflecting Optical Waveguide (ARROW) approach [19], the spectral position of the different transmission windows depend on the well-known normalized frequency (V-parameter) of an isolated high index rod, and depends on its diameter $d$ and the refractive index contrast $\Delta n$ with the low index medium. Theoretically the PBGs can be shifted spectrally by changing only one of these two opto-geometrical parameters, but the confinement and bending losses can be limiting factors [20] as they also depend strongly on the $d / \Lambda$ ratio and $\Delta n$, where $\Lambda$ is the rod-to-rod space and on the micro-structured cladding size relative to the wavelength (proportional to the ratio $\Lambda / \lambda$ ) [18]. The opto-geometrical parameters of the micro-structure need then to be carefully optimised in order to obtain simultaneously low loss and a desired spectral position of the different transmission bands.

To highlight the potential of solid core PBG fibers for supercontinuum (SC) generation [21] toward short wavelengths, we firstly consider a PBG structure with a low index core surrounded by four confinement rings of high index rods (Cargille oil, $n=1.6$ at $\lambda=589.3 \mathrm{~nm}$, step refractive index profile). Pitch and diameter are $3.10 \mu \mathrm{m}$ and $1.23 \mu \mathrm{m}$ respectively. Using a multipole method [22], we calculate the dispersive and non linear guidance properties of the fundamental core guided mode. For both the silica and inclusion the material dispersion is included; we neglect material absorption over the wavelength range considered here. Fig. 2 plots the PBG guidance characteristics obtained as a function of the normalized frequency $V$ of an isolated high index rod. The top panel shows the linear guidance properties: confinement losses (left axis) and group index curves (right axis). The bottom panel plots the effective area (left axis) and the associated non linear parameter $\gamma$ (right axis). In order to illustrate the interest of solid core PBG fibers compared to index guiding PCF, we also plot on Fig. 2 the group index (top panel, dashed line) evolution of the fundamental mode guided into the index guiding PCF with a silica core surrounded by air holes (dimensions as above, with material dispersion).

Due to energy coupling from the core guided mode to cladding modes close to each PBG, the confinement losses, group index and effective area of the core guided mode all undergo dramatic increase contrary to index guiding PCFs. These strong variations of group index curves in solid core PBG fibers close to each bandgap edges can be used to fulfill matching conditions between the trapped dispersive wave and the optical soliton in the infrared.

\section{Group index curve optimization and supercontinuum generation}

In this section we use a finite element method (COMSOL software) to calculate the linear and non linear properties of the fundamental core guided mode for a wider variety of micro-structure configurations considered as mono-modes [23]. The confinement losses are computed using a circular Perfectly Matched Layer (PML) and the material dispersion is always taken into account (silica or doped silica). For the purposes of our simulations, we choose to work with a step refractive index profile of the high index inclusions in the cladding, the core being one missing rod. In order to give general guidelines of group index optimization we work in the first PBG order which is spectrally the widest but also characterized by the highest confinement losses. Section 3.1 considers the effect of varying $\Delta n$ and Section 3.2 considers the effect of varying $d / \Lambda$ Note that the dispersion characteristics depend only weakly on the number of confinement rings considered. On the other hand, the dispersion and non linearity are very strongly affected by the particular opto-geometric parameters of pitch, diameter and $\Delta n$ which are the focus of our study here. In each of these sections we present explicit simulations of the supercontinuum generation dynamics to link the spectral broadening mechanisms with the particular choice of fiber parameters.

\subsection{Varying $\Delta n$}

We plot in Fig. 3 the dependence on wavelength of the (a) effective index, (b) confinement losses, (c) group index $\left(n_{\mathrm{g}}\right)$ and $(d)$ non linear parameter $\gamma$ of the fundamental core guided mode for different microstructure configurations with an increase of $\Delta n$ from 0.01 to 0.1 . The ratio $d / \Lambda$ is fixed to 0.4 in all cases and six confinement rings are simulated. Even if such high refractive index contrasts are impossible to achieve in silica (all solid structures), the infiltration of an air hole PCF with high refractive index fluids can lead to such high $\Delta n$ values [24]. In order to keep the same spectral position of PBG1 (ARROW

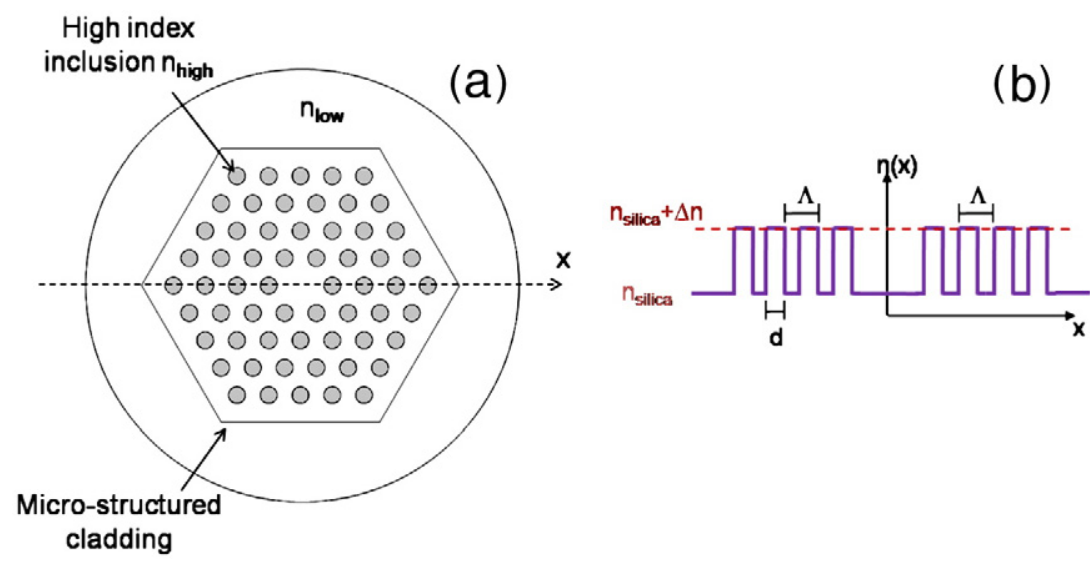

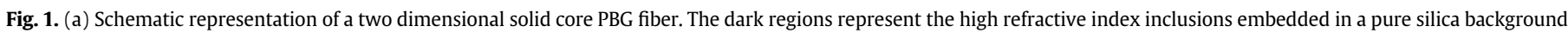
(light regions). (b) Transversal cut showing a step refractive index profile in the inclusions. 


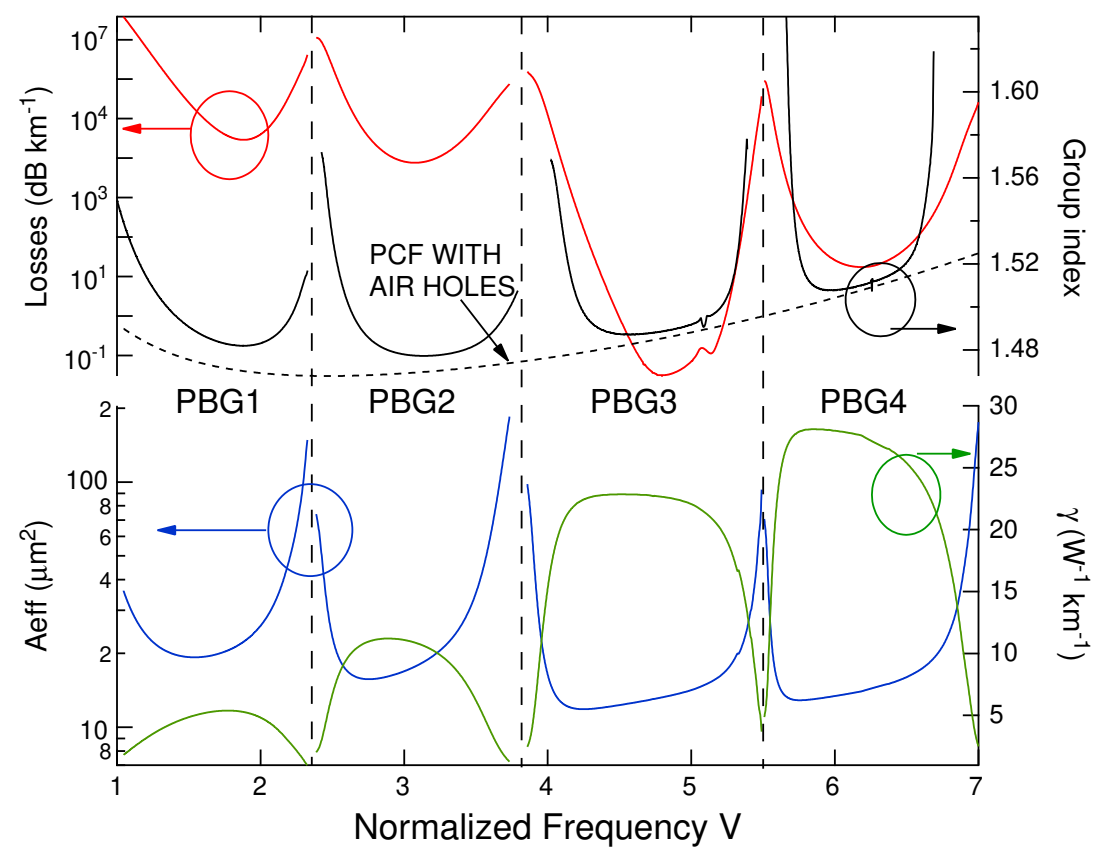

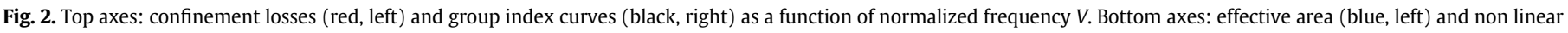

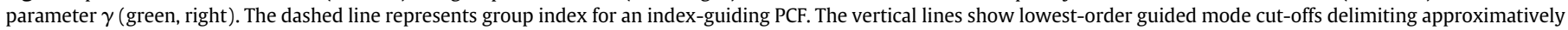
the bandgaps.

model [19]), the rod diameter (and thus $\Lambda$ ) is decreased for each $\Delta n$ value. For example $\Lambda$ goes from $3.9 \mu \mathrm{m}$ to $1.22 \mu \mathrm{m}$.

In Fig. 3(a) and (c), we firstly observe that increasing $\Delta n$ involves a decreasing of the fundamental mode effective index, due to deeper PBG (in effective index) [18]. Consequently, the group index value rises with $\Delta n$ in the center of the PBG, and $n_{\mathrm{g}}$ increases quicker to the long wavelength edge with $\Delta n$. This steep increase of the group index in the infrared is a characteristic of the PBG guidance mechanism and is not observed in the modified total internal reflection PCFs. Moreover we observe that the minimum group index related to the ZDW shifts toward the short wavelength side when increasing $\Delta n$ (from roughly $845 \mathrm{~nm}$ to $570 \mathrm{~nm}$ ).
Fig. 3(b) and (d) show that an increase of $\Delta n$ is followed by a rise of the non linear coefficient and confinement losses over all the PBG. Indeed, when $\Delta n$ increases, $\Lambda$ must be decreased to keep the PBG at the same wavelength. Then the ratio $\Lambda / \lambda$ (size of the micro-structured cladding relative to the wavelength) decreases and leads to a rise of confinement losses and $\gamma$ with $\Delta n$. However (and in contrast to the group index curves variation with $\Delta n$ in Fig. 3(c)), Fig. 3(b) and (d) show that the spectral minima of confinement losses and maxima of non linear parameter in the PBG depend slightly on $\Delta n$. Then for this $d / \Lambda$ value, the shift of the ZDW highlighted in Fig. 3(c) toward the infrared for weak $\Delta n$ is followed by a strong decrease of the light confinement and non linearity. For example at the ZDW the confinement losses go
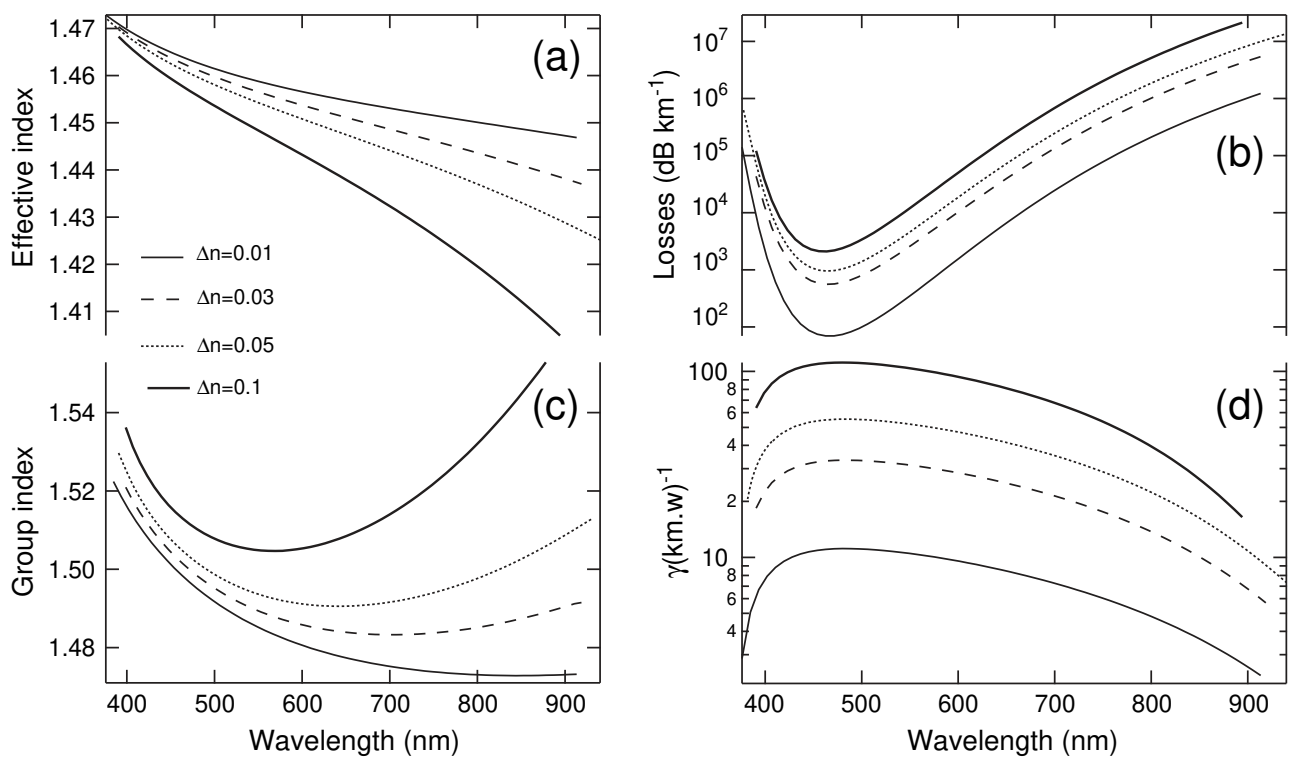

Fig. 3. Spectral evolution of the fundamental mode effective (a) and group index (c), confinement losses (b) and non linear parameter $\gamma$ (d) in PBG1 for different $\Delta n$ values. 
from $2 \times 10^{4} \mathrm{~dB} / \mathrm{km}$ to $4.5 \times 10^{5} \mathrm{~dB} / \mathrm{km}$ when $\Delta n$ decreases from 0.1 to 0.01 and $\gamma$ goes from $100(\mathrm{~W} \mathrm{~km})^{-1}$ to $3.8(\mathrm{~W} \mathrm{~km})^{-1}$.

3.1.1. Supercontinuum generation for fixed soliton order at constant $\beta_{2}$ To model non linear propagation of femtosecond pulses in PBG fibers we use the rigorous frequency-domain approach described by Laesgsgaard [25] using Raman parametrization as in Ref. [26]. This approach allows an approximation-free treatment of the strong frequency-dependent loss, dispersion and non linearity of PBG fibers [17]. To highlight the $\Delta n$ influence on the short wavelength generation, we simulate non linear spectral broadening in PBG1 in the different structures presented in Fig. 3 for a constant soliton order $N^{2}=\gamma P_{0} T_{0}^{2} /\left|\beta_{2}\right|$. For each set of parameter, the pump wavelength is changed so that the GVD is constant at $\beta_{2}=-10 \mathrm{ps}^{2} / \mathrm{km}$ (anomalous regime). The temporal pulse width being held constant at $50 \mathrm{fs}$, the input peak power $P_{0}$ is changed to keep the product $P_{0} \gamma$ constant at the pump wavelength $(N \simeq 10)$. The computed non linear length at the pump is close to $0.7 \mathrm{~mm}$ for all the cases.

For the lowest value of $\Delta n(0.01)$ and for a $d / \Lambda$ of 0.4 , Fig. 3(c) shows that the increase of $n_{\mathrm{g}}$ to the infrared is not sufficient to generate a trapped dispersive wave on the blue side before reaching high confinement losses close to the PBG edge. Fig. 4 shows then simulated SC evolutions for the three different $\Delta n$ values 0.1 (a), 0.05 (b) and 0.03 (c). We observe in Fig. 4(a) $\left(P_{0} \sim 16.5 \mathrm{~kW}\right)$ that a coupling between the soliton and the phase matched dispersive wave (around $450 \mathrm{~nm}$ ) occurs around the first millimetres of propagation while the first ejected optical soliton is largely attenuated after a propagation distance of $10 \mathrm{~cm}$. When $\Delta n$ decreases, Fig. 4(b) and (c) show a shift of the whole SC toward the infrared where losses are higher. Consequently the first ejected optical soliton is attenuated over a shorter distance (around $4 \mathrm{~cm}$ and $1 \mathrm{~cm}$ for
$\Delta n=0.05$ and 0.03 respectively) and the coupling efficiency with the red shifted dispersive wave is limited. Moreover small $\Delta n$ values involve higher input peak power $\left(\mathrm{P}_{0} \sim 100 \mathrm{~kW}\right.$ for $\left.\Delta n=0.03\right)$ due to reduced non linearity.

\subsubsection{Supercontinuum generation for a fixed input pulse wavelength}

In this section we propose to highlight the $\Delta n$ influence on the non linear spectral broadening when the input pulse wavelength is fixed. As in section 3.1.1 the soliton order at the pump wavelength is kept constant through the ratio $P_{0} \gamma /\left|\beta_{2}\right|(N \simeq 8)$. Fig. 5 shows then the supercontinuum evolution over $20 \mathrm{~cm}$ of fiber when pumped at $650 \mathrm{~nm}$ for the different $\Delta n$ values: 0.03 (a), 0.05 (b) and 0.1 (c).

By changing $\Delta n$ and keeping the same input pulse wavelength, we modify $\beta_{2}$ and the GVD regime. For $\Delta n=0.1, \beta_{2}$ is negative at $650 \mathrm{~nm}$ ( $-20 \mathrm{mps}^{2} / \mathrm{km}$, anomalous regime). Fig. 5(a) shows the non linear spectral broadening occurring for an input peak power of $20 \mathrm{~kW}$. We clearly observe after a few millimetres of propagation the generation of a dispersive wave around $400 \mathrm{~nm}$ phase matched with optical soliton propagating in the infrared. When $\Delta n=0.05, \beta_{2}$ is very weak and close to the ZDW $\left(\sim-1 \mathrm{ps}^{2} / \mathrm{km}\right)$. Because the required input peak power is low at $\sim 2.3 \mathrm{~kW}$, Fig. 5 (b) does not show non linear spectral broadening. When $\Delta n$ decreases to 0.03 , the GVD regime becomes normal $\left(\beta_{2} \sim+5.5 \mathrm{ps}^{2} / \mathrm{km}\right.$ at $650 \mathrm{~nm}$ and $\left.\mathrm{P}_{0} \sim 18 \mathrm{~kW}\right)$. Fig. 5 (c) shows the limited spectral broadening due to self phase modulation. In this regime the non linear processes are not dominated by soliton fission and dispersive waves are not generated on the short wavelength side. Note that the conversion efficiency from pump to short wavelength dispersive wave is here comparable to that seen in the standard index guiding PCF $(<1 \%)$ but with the strong pump powers used, useful intensities of short wavelength light can nonetheless be generated.
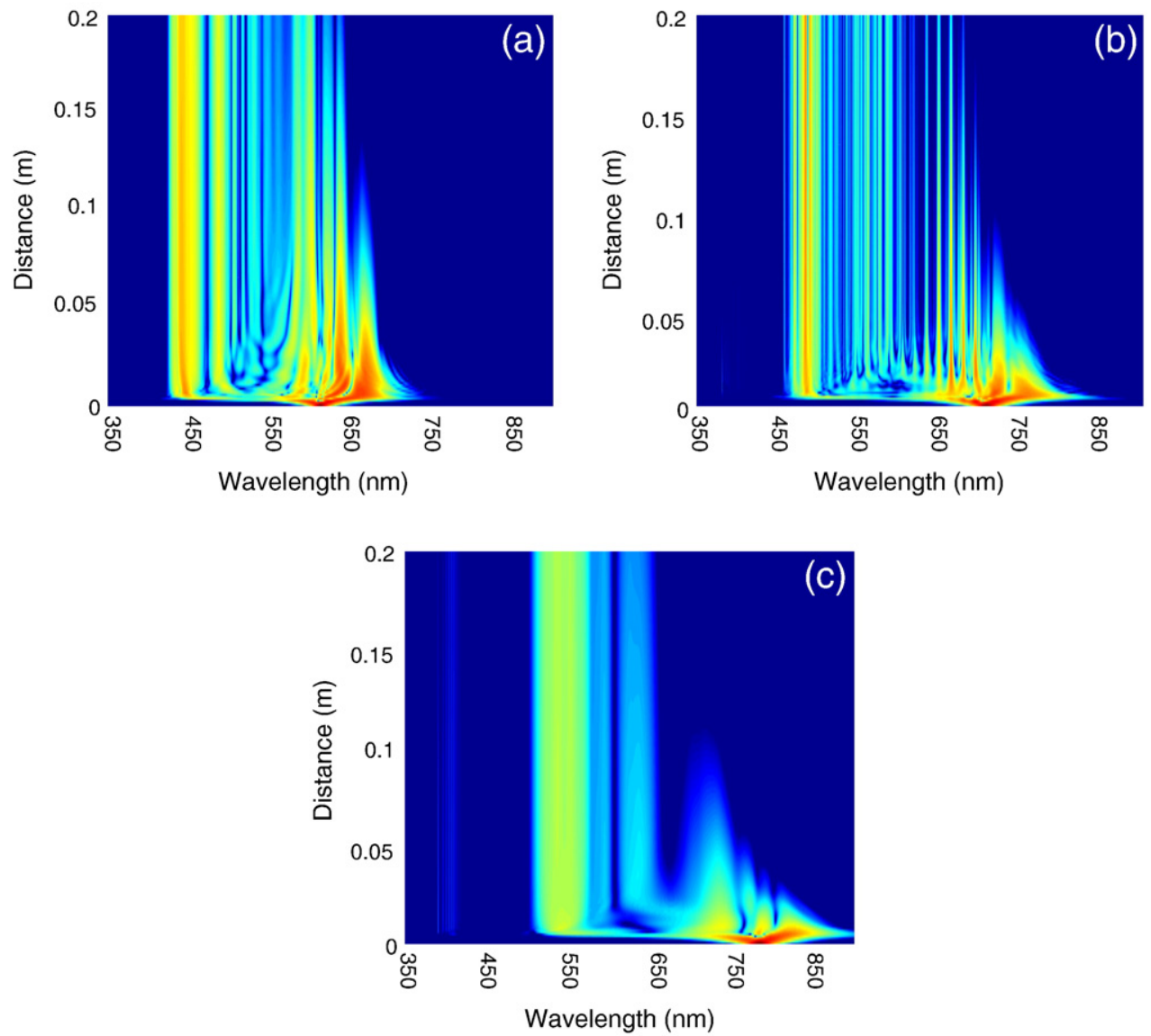

Fig. 4. Simulated SC evolutions in PBG1 over a fiber length of $20 \mathrm{~cm}$ for $\Delta n=0.1$ (a), 0.05 (b) and 0.03 (c). $\beta_{2}$ is fixed at $-10 \mathrm{ps}{ }^{2} / \mathrm{km}$. 

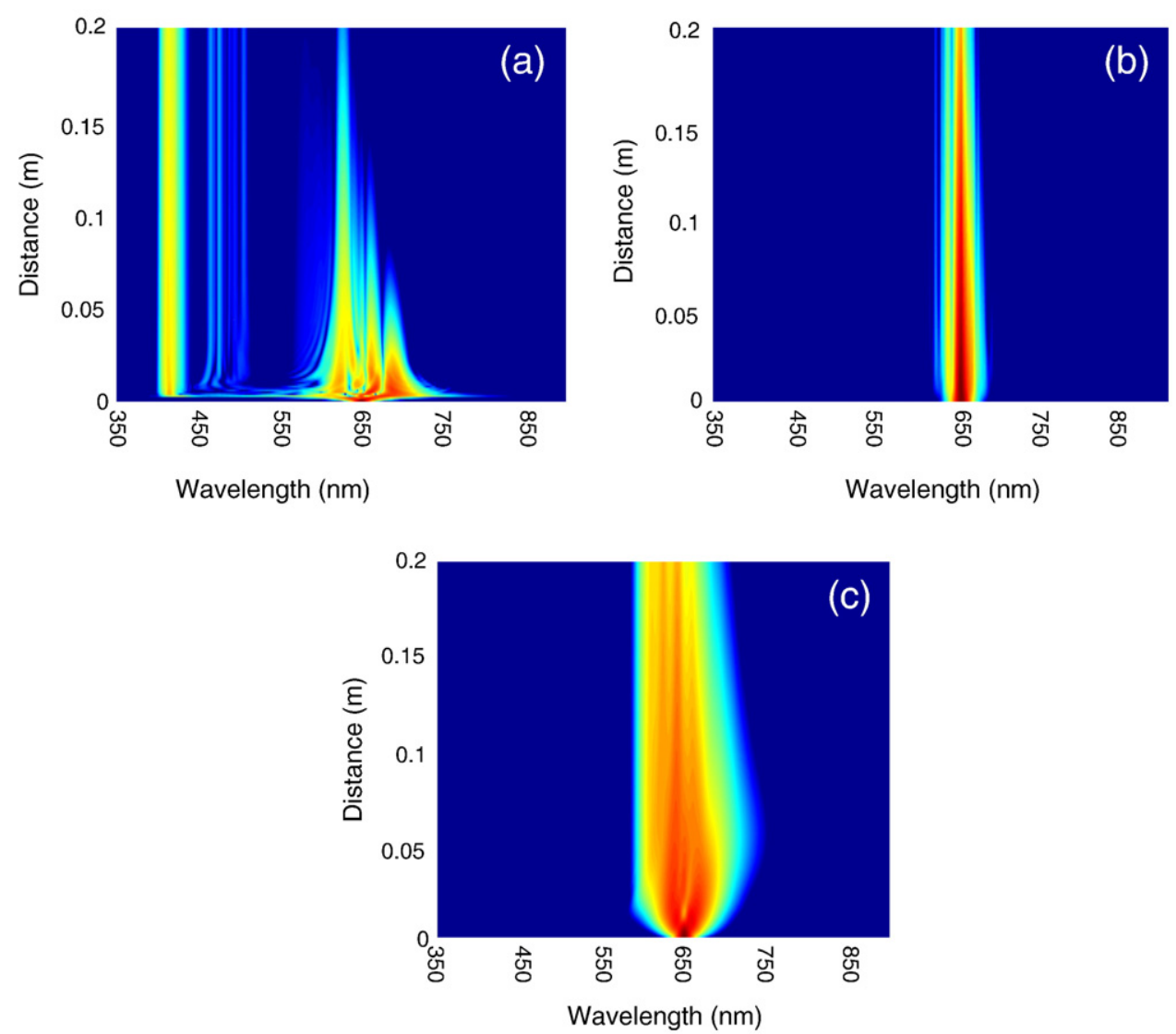

Fig. 5. Simulated SC evolutions in PBG1 over a fiber length of $20 \mathrm{~cm}$ for $\Delta n=0.1$ (a), 0.05 (b) and 0.03 (c). The input pulse wavelength is fixed at 650 nm.

\subsection{Varying $d / \Lambda$}

We plot in Fig. 6 the effective (a) and group index (c) variations of the fundamental core guided mode, its confinement losses (b) and its non linear parameter $\gamma(d)$ versus wavelength in the first PBG for the different micro-structure configurations with $d / \Lambda$ ratios of $0.2,0.4$ and 0.6 . The refractive index contrast $\Delta n$ is set to 0.03 in all cases and seven confinement rings are simulated. As the spectral position of the transmission windows depends only on $d$ and $\Delta n$ [19], only the pitch of the micro-structure is changed from $4.5 \mu \mathrm{m}$ to $1.5 \mu \mathrm{m}$. In Fig. 6(a) and (c), we observe that increasing the ratio $d / \Lambda$ leads to a decrease of the fundamental mode effective index, due to deeper PBG [18]. Consequently the group index value rise with $d / \Lambda$ around the center of the PBG and $n_{\mathrm{g}}$ increases quicker with $d / \Lambda$ in the infrared. Moreover, the ZDW shifts toward the short wavelength when increasing $d / \Lambda$ (from roughly $895 \mathrm{~nm}$ to $600 \mathrm{~nm}$ ).

Fig. 6(b) and (d) show that an increase of $d / \Lambda$ is followed by an increase of $\gamma$ (around the center of the PBG) and of the confinement
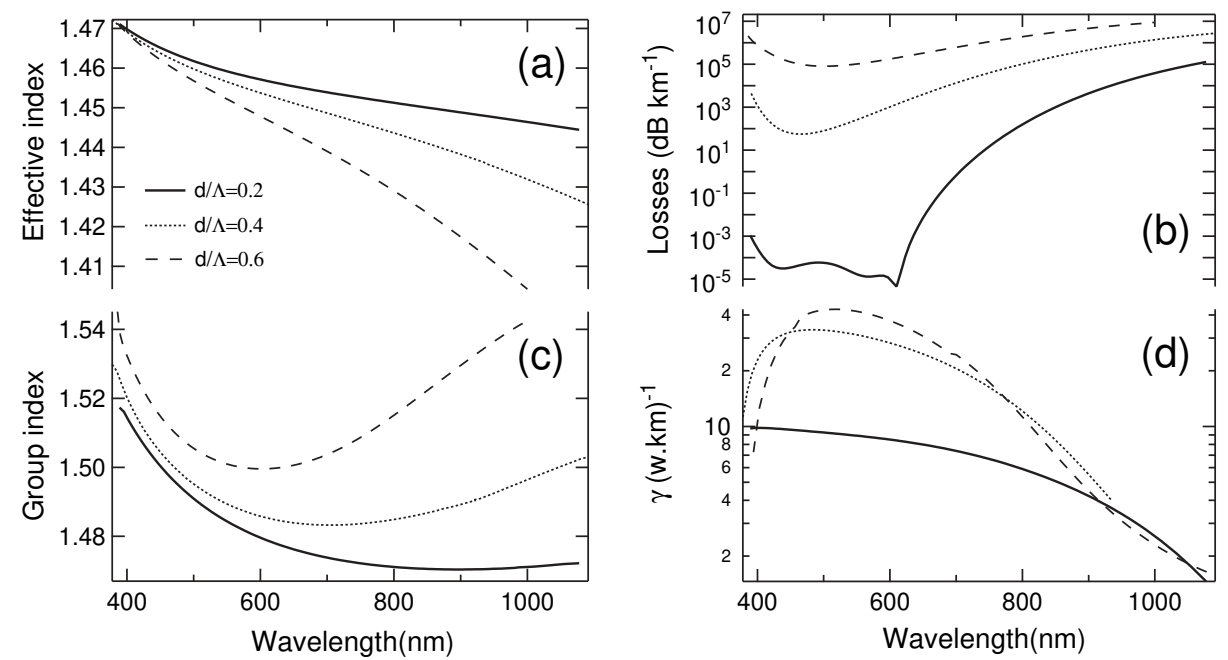

Fig. 6. Spectral evolution of the fundamental mode effective (a) and group index (c), confinement losses (b) and non linear parameter $\gamma(\mathrm{d})$ in PBG1 for different $d / \Lambda$ ratio. 
losses. Indeed, the rise of the $d / \Lambda$ ratio involves a decrease of $\Lambda$ ( $d$ being constant). The ratio $\Lambda / \lambda$ decreases and leads to an increase of confinement losses and $\gamma$ with $d / \Lambda$. For the smallest $d / \Lambda$ ratio, the high index inclusions are widely spaced apart. The confinement losses characteristics of the core guided mode correlate with the scattering properties of a single cylinder which are Fano resonance [27]. Due to these properties the confinement losses of the fundamental mode in the first PBG can be very low in the center of the transmission band even with a few rings. Fig. 6(b) and (d) highlight moreover that the loss minimum and non linear parameter maximum shift slightly toward long wavelength when increasing $d / \Lambda$ (comparing 0.4 and 0.6 ) whereas the $d / \Lambda=0.2$ case is not comparable regarding the loss.

\subsubsection{Supercontinuum generation for a fixed $\beta_{2}$}

We simulate supercontinuum generation in PBG1 for the different $d / \Lambda$ presented in Fig. 6 and for a constant soliton order. Again the pump wavelength is changed such that $\beta_{2}$ is fixed to $-10 \mathrm{ps}^{2} / \mathrm{km}$ (anomalous regime) and the input peak power $P_{0}$ is changed to keep the product $P_{0} \gamma$ constant at the pump wavelength $(N \simeq 5)$.

Fig. 7 shows the simulated SC evolution over $20 \mathrm{~cm}$. We observe that the shift in the pump wavelength associated with maintaining constant $\beta_{2}$ and with increasing $d / \Lambda$ leads to a shift of the generated SC toward the short wavelength side as the ZDW decreases $\left(P_{0}=100 \mathrm{~kW}\right.$ for $d / \Lambda=0.2$ ). Consequently the generated DW phase matched with the optical soliton reaches shorter wavelength with a high $d / \Lambda$ value, but the SC becomes spectrally narrower according to the " $U$ " shaped form of the group index presented in Fig. 6(c). Moreover the shift of the ZDW to short wavelength when $d / \Lambda$ increases enables propagation in a regime of low confinement losses as the loss minimum is closer to the short wavelength side in PBG1. The required input peak power is also reduced at $\sim 10 \mathrm{~kW}$ for $d / \Lambda=0.6$ because of the higher non linear parameter. However for $d / \Lambda=0.6$ (Fig. $7(\mathrm{c})$ ) the confinement losses are very high both at the dispersive wave and the optical soliton wavelengths and thus the coupled energy to the visible decreases rapidly with fiber length.

\subsubsection{Supercontinuum generation for a fixed input pulse wavelength}

In this section we investigate the $d / \Lambda$ influence on the non linear spectral broadening when the input wavelength is fixed. As in the previous section the soliton order is constant at the pump wavelength through the ratio $\mathrm{P}_{0} \gamma /\left|\beta_{2}\right|(N \simeq 5)$. The simulated structures shown in Figs. 6 and 8 represent the SC evolutions when pumped at $750 \mathrm{~nm}$ for the different $d / \Lambda$ values: 0.2 (a), 0.4 (b) and 0.6 (c).

As the ZDW changes with the ratio $d / \Lambda$, by keeping the input pulse wavelength constant we modify the GVD regime. For $d / \Lambda=0.2, \beta_{2}$ is positive at $750 \mathrm{~nm}\left(\sim+26 \mathrm{ps}^{2} / \mathrm{km}\right)$. In the normal GVD regime Fig. 8 (a) shows the initial and symmetric non linear spectral broadening mainly due to self phase modulation. Optical solitons are not generated in the normal regime and neither DW in short wavelengths. For $d / \Lambda=0.4, \beta_{2}$ is close to the ZDW and in the anomalous GVD regime $\left(\sim-19 \mathrm{ps}^{2} / \mathrm{km}\right)$. Fig. 8(b) put in evidence generation of optical soliton phase matched with DW around $550 \mathrm{~nm}$. When $\mathrm{d} / \Lambda=0.6, \beta_{2}$ decreases to $\sim-116 \mathrm{ps}^{2} / \mathrm{km}$. Whereas DW generation at shorter wavelengths is expected, Fig. 8(c) shows that the level of losses around $750 \mathrm{~nm}$ is too high and prevent non linear spectral broadening and soliton fission even after a few millimetres.
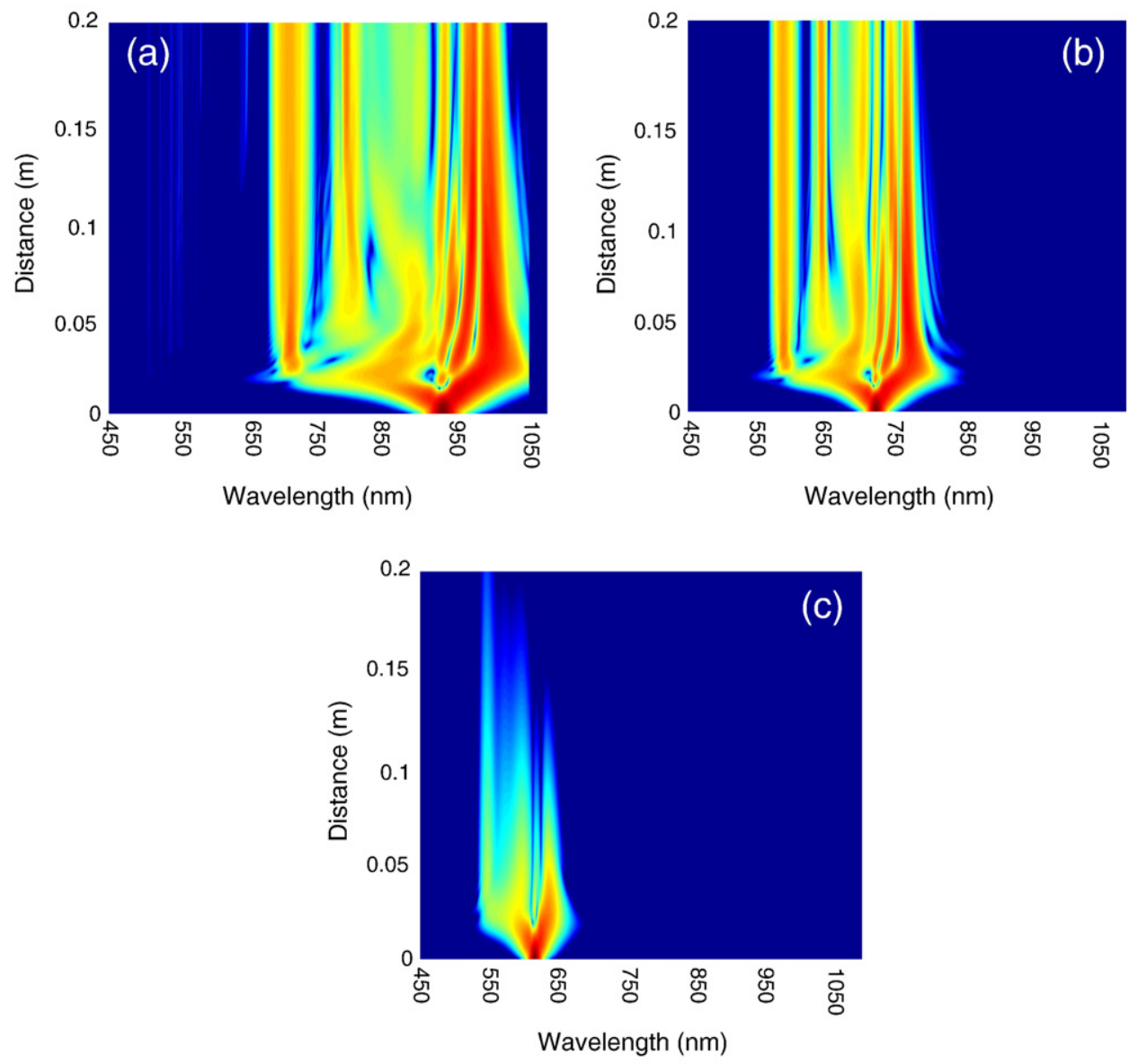

Fig. 7. Simulated SC evolutions in PBG1 over a fiber length of $20 \mathrm{~cm}$ for $d / \Lambda=0.2$ (a), 0.4 (b) and 0.6 (c). $\beta_{2}$ is fixed at $-10 \mathrm{ps} / \mathrm{km}$. 

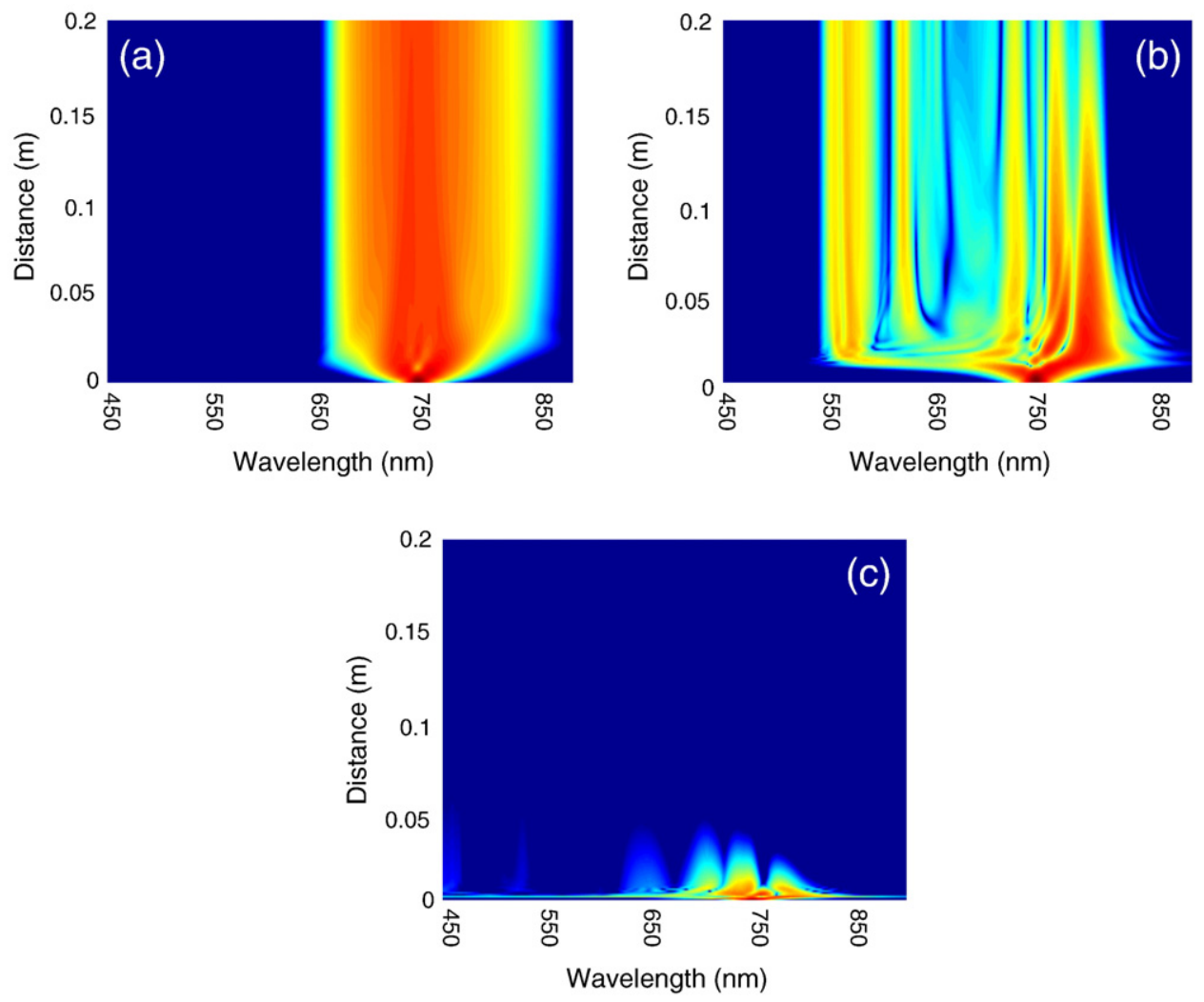

Fig. 8. Simulated SC evolutions in PBG1 over a fiber length of $20 \mathrm{~cm}$ for $d / \Lambda=0.2$ (a), 0.4 (b) and 0.6 (c). The input pulse wavelength is fixed at $750 \mathrm{~nm}$.

\subsection{Discussion}

Numerical results of Sections 3.1 and 3.2 show that when the PBG become deeper in effective index, increasing for example the refractive index contrast $\Delta n$ and/or the ratio $d / \Lambda$ of the micro-structured cladding, the group index curve of the fundamental core guided mode rises mainly on the long wavelength side, while the short wavelength remains dominated by material dispersion in the spectral range of interest. Figs. 3(c) and 6 (c) show that the frequency dependence of group index can be stronger than in index guiding PCFs and highlight for the first time the possibilities of group index matching without reaching a soliton wavelength above $1 \mu \mathrm{m}$ in the infrared.

Additionally Figs. 3(b) and 6 (c) show that such high values of $\Delta n$ and $d / \Lambda$ involve lower ZDW and high confinement losses that can represent a limiting factor. Indeed the results highlighted in Sections 3.1 and 3.2 show that the " $U$ " form of the group index can improve the shift of the generated dispersive wave toward the blue for high $\Delta n$ and/or $d / \Lambda$ values, but the losses prevent the optical soliton propagation in the infrared and thus limit the coupled energy to the dispersive wave. Consequently a compromise must be found between rising of the group index in the infrared and weak losses. A careful design must be undertaken to decrease the losses in the fundamental bandgap by increasing for example the number of confinement rings. The adding of an external ring of air holes around the micro-structured cladding [28] or interstitial air holes between each rod [29] is well known to reduce strongly both confinement and bending losses. With sufficient low losses the use of solid core PBG fibers can lead to the generation of short wavelength components without reaching wavelength above $1 \mu \mathrm{m}$ in the infrared.

\section{Energy transfer across different PBG orders}

In this section we propose to demonstrate numerically short wavelengths generation in the blue pumping into the anomalous GVD of a typical solid core PBG structure in the femtosecond regime. In order to highlight the potential of such PBG fibers on the non linear spectral broadening of ultra-short pulses, we choose to generate a dispersive wave across a spectral region of high attenuation pumping into an adjacent PBG [17].

We consider a PBG structure with a low index core (pure silica) surrounded by four confinement rings of high index rods (Cargille oil, $n=1.6$ at $\lambda=589.3 \mathrm{~nm}$ ). Pitch and $d / \Lambda$ ratio are $2.44 \mu \mathrm{m}$ and $\sim 0.4$ respectively. All the linear and non linear optical properties of the fundamental core guided mode are simulated using the multipole method (with material dispersion) [22]. We plot in Fig. 9 the group

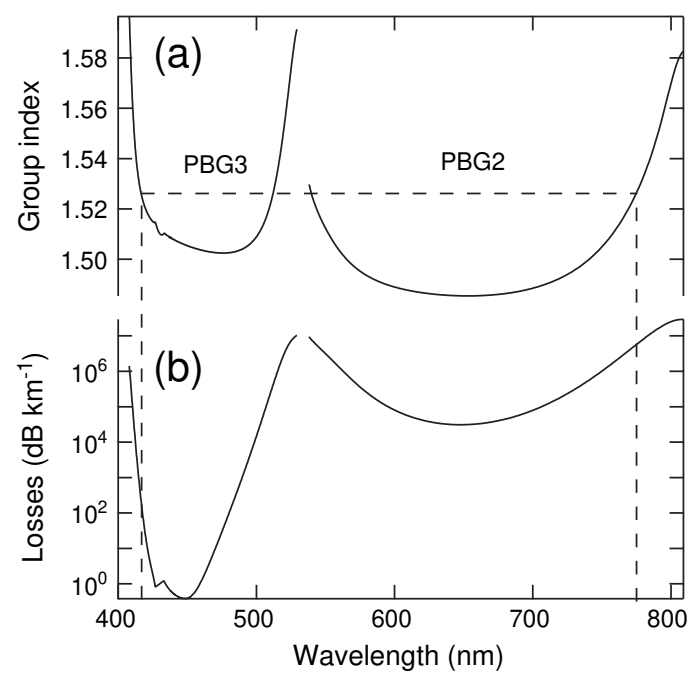

Fig. 9. Spectral evolution of the group index (a) and confinement losses (b) of the fundamental core mode within the 2nd and 3rd PBG order. 


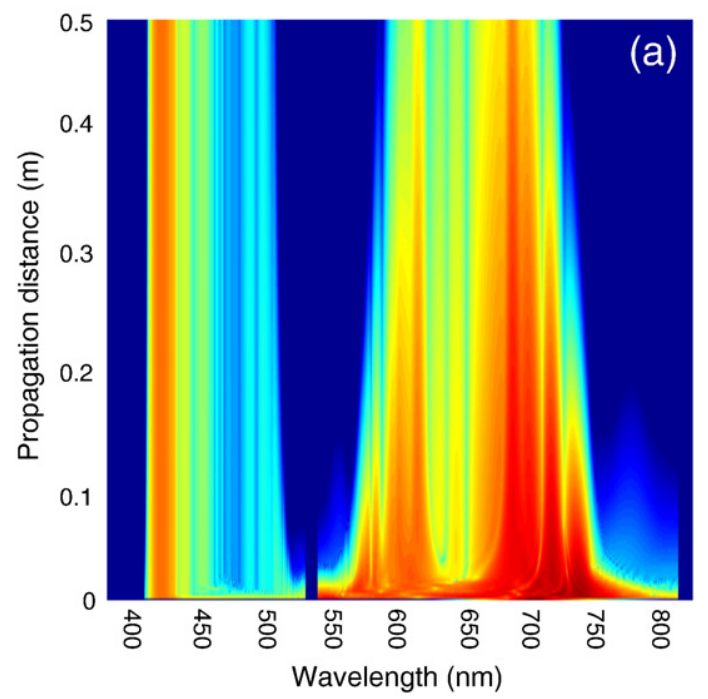

(b)

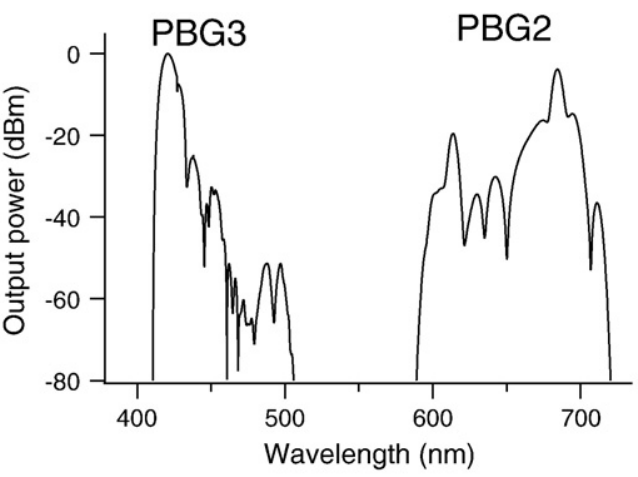

Fig. 10. (a) SC evolution in PBG2 and PBG3 over fiber length of $50 \mathrm{~cm}\left(P_{\text {in }}=100 \mathrm{~kW}, \lambda_{\mathrm{pump}}=700 \mathrm{~nm}\right)$. (b) Normalized output spectrum after $50 \mathrm{~cm}$ of propagation.

index and confinement losses spectral variations of this mode confined within the second and third PBG. We observe potential group index matching (dashed lines) between the long wavelength side of the 2nd PBG (where the optical soliton propagates when pumping into its anomalous GVD regime) and the blue edge of PBG3 around $420 \mathrm{~nm}$ where a dispersive wave can be generated.

Pumping at $700 \mathrm{~nm}$ with an input peak power of $100 \mathrm{~kW}$ and $50 \mathrm{fs}$ pulse duration (anomalous GVD regime), Fig. 10 highlights the energy transfer from the first ejected optical soliton propagating in PBG2 to a dispersive wave generated in the blue side of PBG3. Even if the fundamental soliton in PBG2 is attenuated after a propagation distance of $20 \mathrm{~cm}$, the energy transfer occurs over the first millimetres of propagation because the computed non linear length at the pump is close to $0.4 \mathrm{~mm}$. Then once generated in the short wavelength in low loss regions of PBG3 the dispersive wave see much lower attenuation and can propagate over a longer distance. Fig. 10(b) plots the normalized output spectrum simulated after a propagation distance of $50 \mathrm{~cm}$. To the best of our knowledge this is the first demonstration of the possibility of such short wavelength generation in PBG fibers. As the spectral position of the different transmission bands can be shifted by varying the rod diameter or the refractive index contrast $\Delta n$ of the micro-structured cladding, a slight reduction of the rod-to-rod space ( $d / \Lambda$ fixed) or $\Delta n$ (using another oil) can shift the generated dispersive wave below $400 \mathrm{~nm}$. This shift of the bands toward the blue is also associated with an increase of fundamental mode confinement losses which can be decreased by increasing the number of confinement rings for example.

\section{Conclusion}

In this paper, we have studied numerically the use of solid core photonic bandgap fibers for visible supercontinuum generation. The strong frequency-dependent group index curve of the fundamental mode close to the bandgap edge allows dispersive wave generation in short wavelengths without the need to reach soliton wavelengths above $1 \mu \mathrm{m}$ as is the case with modified total internal reflection PCF. We numerically investigate the impact of opto-geometrical parameters of the micro-structured cladding on supercontinuum generation in the visible, such as the rod-to-rod space and the refractive index contrast between the inclusion and the low index medium. Design criteria are highlighted to generate efficiently visible wavelength which have to be associated with sufficient low confinement losses. Moreover taking into account all the strong frequency-dependent optical properties of such structures we demonstrate the generation of short wavelengths on the blue edge of a bandgap pumping into its adjacent bandgap through a spectral region of high attenuation.

\section{Acknowledgment}

Authors thank M. Abdilahi for the helpful discussions. We acknowledge support from the French Agence Nationale de la Recherche projects MANUREVA ANR-08-SYSC-019 and IMFINI ANR-09-BLAN-0065.

\section{References}

[1] J.K. Ranka, R.S. Windeler, A.J. Stentz, Opt. Lett. 25 (2000) 25.

[2] S. Coen, A.H.L. Chau, R. Leonhardt, J.D. Harvey, J.C. Knight, W.J. Wadsworth, P.St.J. Russell, Opt. Lett. 26 (2001) 1356.

[3] T.A. Birks, W.J. Wadsworth, P.St.J. Russell, Opt. Lett. 25 (2000) 1415.

[4] J.M. Dudley, G. Genty, S. Coen, Rev. Mod. Phys. 78 (2006) 1135.

[5] J.M. Dudley, J.R. Taylor, Nat. Photonics 3 (2009) 85.

[6] J.C. Knight, T. Birks, P. Russell, D. Atkin, Opt. Lett. 21 (1996) 1547.

[7] G. Genty, M. Lehtonen, H. Ludvigsen, Opt. Express 12 (2004) 4614.

[8] A.V. Gorbach, D.V. Skryabin, J.M. Stone, J.C. Knight, Opt. Express 14 (2006) 9854

[9] A.V. Gorbach, D.V. Skryabin, Nat. Photonics 1 (2007) 653

[10] J.C. Travers, A.B. Rulkov, B.A. Cumberland, S.V. Popov, J.R. Taylor, Opt. Express 16 (2008) 14435.

[11] A. Kudlinski, A.K. George, J.C. Knight, J.C. Travers, A.B. Rulkov, S.V. Popov, J.R. Taylor, Opt. Express 14 (2006) 5715.

[12] A. Kudlinski, A. Mussot, Opt. Lett. 33 (2008) 2407.

[13] J.M. Stone, J.C. Knight, Opt. Express 16 (2008) 2670.

[14] A. Kudlinski, G. Bouwmans, M. Douay, M. Taki, A. Mussot, J. Lightwave Technol. 27 (2009) 1556.

[15] W.J. Wadsworth, N. Joly, J.C. Knight, T.A. Birks, F. Biancalana, P.St.J. Russell, Opt Express 12 (2004) 299.

[16] A. Bétourné, A. Kudlinski, G. Bouwmans, O. Vancincq, A. Mussot, Y. Quiquempois, Opt. Lett. 34 (2009) 3083.

[17] V. Pureur, J.M. Dudley, Opt. Lett. 15 (2010) 2813.

[18] V. Pureur, A. Bétourné, G. Bouwmans, L. Bigot, A. Kudlinski, K. Delplace, A. Le Rouge, Y. Quiquempois, M. Douay, Fiber Integr. Opt. 28 (2009) 27.

[19] N.M. Litchinitser, S.C. Dunn, B. Usner, B.J. Eggleton, T.P. White, R.C. McPhedran, C.M. De Sterke, Opt. Express 11 (2003) 1243.

[20] T.A. Birks, F. Luan, G.J. Pearce, A. Wang, J.C. Knight, D.M. Bird, Opt. Express 14 (2006) 5688.

[21] A. Fuerbach, P. Steinvurzel, J.A. Bolger, A. Nulsen, B.J. Eggleton, Opt. Lett. 30 (2005) 830.

[22] T.P. White, B.T. Kuhlmey, R.C. McPhedran, D. Maystre, G. Renversez, C.M. De Sterke, L.C. Botten, JOSA B 19 (2002) 2322.

[23] V. Pureur, J.C. Knight, B.T. Kuhlmey, Opt. Express 18 (2010) 8906.

[24] B.T. Kuhlmey, B.J. Eggleton, D.K.C. Wu, J. Lightwave Technol. 27 (2009) 1617.

[25] J. Laegsgaard, Opt. Express 15 (2007) 16110.

[26] G.P. Agrawal, Nonlinear Fiber Optics, Academic Press, San Diego, 2007.

[27] P. Steinvurzel, C. Martijn de Sterke, M.J. Steel, B.T. Kuhlmey, B.J. Eggleton, Opt Express 14 (2006) 8797

[28] A. Bétourné, V. Pureur, G. Bouwmans, Y. Quiquempois, L. Bigot, M. Perrin, M. Douay, Opt. Express 15 (2007) 316.

[29] A. Bétourné, G. Bouwmans, Y. Quiquempois, M. Perrin, M. Douay, Opt. Lett. 32 (2007) 1719. 